University of Nebraska - Lincoln

DigitalCommons@University of Nebraska - Lincoln

Virology Papers

Virology, Nebraska Center for

$12-1-1992$

\title{
Processing and Localization of Dengue Virus Type 2 Polyprotein Precursor NS3-NS4A-NS4B-NS5
}

\author{
Luwen Zhang \\ University of Nebraska-Lincoln, Izhang2@unl.edu \\ P. Maruthi Mohan \\ University of Kansas Medical Center \\ R. Padmanabhan \\ University of Kansas Medical Center
}

Follow this and additional works at: https://digitalcommons.unl.edu/virologypub

Part of the Virology Commons

Zhang, Luwen; Mohan, P. Maruthi; and Padmanabhan, R., "Processing and Localization of Dengue Virus Type 2 Polyprotein Precursor NS3-NS4A-NS4B-NS5" (1992). Virology Papers. 12.

https://digitalcommons.unl.edu/virologypub/12

This Article is brought to you for free and open access by the Virology, Nebraska Center for at DigitalCommons@University of Nebraska - Lincoln. It has been accepted for inclusion in Virology Papers by an authorized administrator of DigitalCommons@University of Nebraska - Lincoln. 


\title{
Processing and Localization of Dengue Virus Type 2 Polyprotein Precursor NS3-NS4A-NS4B-NS5
}

\author{
LUWEN ZHANG, P. MARUTHI MOHAN, $\dagger$ AND R. PADMANABHAN* \\ Department of Biochemistry and Molecular Biology, University of Kansas Medical Center \\ Kansas City, Kansas 66103
}

Received 2 April 1992/Accepted 28 August 1992

\begin{abstract}
Processing of dengue virus type 2 polyprotein precursor NS3-NS4A-NS4B-NS5 could be mediated by the catalytically active NS3 protease domain and NS2B in trans at the dibasic sites NS3-NS4A and NS4B-NS5. Subcellular localization of the unprocessed precursor NS3-NS4A-NS4B-NS5 showed that it was confined to a distinct subcellular organelle in the cytoplasm, which was distinct from the distribution of the mature NS5.
\end{abstract}

Dengue virus type 2 (DEN-2), a member of the family Flaviviridae, contains a single-stranded RNA genome of 10,723 nucleotides (New Guinea $C$ strain), having a type 1 cap at the $5^{\prime}$ end, but lacking a poly(A) tract at the $3^{\prime}$ end (for reviews, see references $1,4,23,27$, and 28 ). The genomic RNA is of positive-strand polarity, having a single open reading frame that encodes a polyprotein of 3,391 amino acids, which is processed into three structural and at least seven nonstructural proteins so far identified. The gene order is 5'-C-prM-E-NS1-NS2A-NS2B-NS3-NS4A-NS4BNS5-3', where C, prM, and E are the structural proteins and NS1 through NS5 represent the nonstructural proteins. The processing of the polyprotein precursor occurs cotranslationally as well as posttranslationally and is performed by either the host signalase in association with the membranes of the endoplasmic reticulum or the viral protease(s) (reviewed in reference 4). For the construction of expression plasmids to study the processing of the DEN-2 polyprotein precursors, a recombinant vaccinia virus expression vector (pTM1) was used (18). This vector contains a T7 promoter $\left(\mathrm{P}_{\mathrm{T7}}\right)$ and an encephalomyocarditis virus (EMCV) 5'-untranslated region upstream of the target gene, which allows cap-independent translation of the transcripts; with this system, the target gene has been reported to be expressed at high levels of RNA as well as protein because of more efficient utilization of the transcripts by cap-independent translation $(7,19)$. Figure 1 shows the polyprotein precursor of DEN-2 and the location of the coding sequences of the nonstructural proteins used in this study. These expression plasmids were constructed by standard recombinant DNA techniques (17) as follows. The details of the construction of the intermediate plasmids pLZ-3PCR, pLZ-3, pLZ-5, pLZ123 , and pLZ-2B-3-4A-4B-5 and the plasmid pLZ-12345 encoding all the nonstructural proteins of DEN-2 will be described elsewhere (unpublished data).

pLZ-345. The pLZ-3PCR plasmid was digested with NsiI (nucleotide 4696) and EcoRI in the pTM1 vector and then ligated with two other DNA fragments; one of these was obtained from the plasmid pLZ-3 by digesting with NsiI and PvuII (nucleotides 4696 to 5447), and the other was from the cDNA clone pPM-A10 (14) by digesting with PvuII and EcoRI (nucleotides 5447 to 8761 ). This cloning procedure

\footnotetext{
* Corresponding author.

† Present address: Department of Biochemistry, Osmania University, Hyderabad-500007, (A.P.), India.
}

resulted in the plasmid pLZ-310 containing the coding sequence NS3-NS4A-NS4B-truncated NS5. pLZ-310 was then digested with StuI to release a 900 -bp fragment between nucleotide 7872 and a site in the vector. This fragment was replaced by the StuI fragment $(2.7 \mathrm{~kb})$ from pLZ-5, and the plasmid pLZ-345 was obtained containing the region from NS3 to NS5 (nucleotides 4522 to 10543).

pLZ-2B. A polymerase chain reaction (PCR) was performed with two oligonucleotides, 5'-AGCTGGCCACTAA ATGAGGCT-3' (nucleotides 4132 to 4152 ) and 5'-ttaCCGTT GTTTCTTCACTTCCCACAG-3' (complementary to nucleotides 4498 to 4521 ), on the pLZ-123 template, and the PCR fragment was cloned into the PTM1 vector at the NcoI site to obtain the pLZ-2B plasmid containing the complete NS2B coding sequence and ending with the termination codon TAA (tta in the oligonucleotide).

pLZ-2B3(pro) and pLZ-3(pro). The plasmid pLZ-2B345 was digested with $X h o I$ (nucleotide 5426) and $X b a I$ (in the pTM1 vector) and cloned into the pTM1 vector digested with $X b a I$ and $X h o I$ to obtain the plasmid pLZ-2B3(pro) encoding the complete NS2B and the N-terminal region of NS3 $(\sim 50 \%)$. pLZ-3(pro) was constructed by a procedure similar to the procedure followed for pLZ-2B3(pro), but using pLZ-3 instead of the pLZ-2B345 plasmid.

pLZ-3(50\%C)45. pLZ-345 was digested with XhoI (nucleotide 5426) and $X b a I$ (in the pTM1 vector), and the large fragment containing the C-terminal NS3(50\%)45 sequences was ligated with the small $X b a I-X h o I$ fragment obtained from the pTM1 vector; this step gave rise to the plasmid pLZ-3 $(50 \% \mathrm{C}) 45$. The $\mathbf{N}$ terminus of the clone contained 13 amino acid residues from the multiple cloning sites of the pTM-1 vector.

pLZ-3*45. Two oligonucleotides, 5'-GCTGGAGTATTGT GGGAT-3' (nucleotides 4522 to 4539 ) and 5'-TTATGCAT TAGAACAGCGCCGCGTGTGACAGCCCACATTGTATT$3^{\prime}$ (complementary to nucleotides 4660 to 4703 ), were used for PCR; the longer one contained the desired mutation converting histidine into alanine in the active-site catalytic triad (as shown by an underline); an additional mutation (as shown by an overline) was found to be present in three independent clones, which resulted in the His $\rightarrow$ Asn change; this mutation may have resulted from the $G \rightarrow T$ change at the 3 '-terminal nucleotide of the oligonucleotide primer used for PCR. The resulting mutant PCR fragment (nucleotides 4522 to 4703 ) was cloned into the PTM1 vector at the NcoI site. The resulting plasmid, pLZ-3*PCR, was digested with $N$ siI (nucleotide 4696) and $X b a I$ and ligated with the NsiI-XbaI 


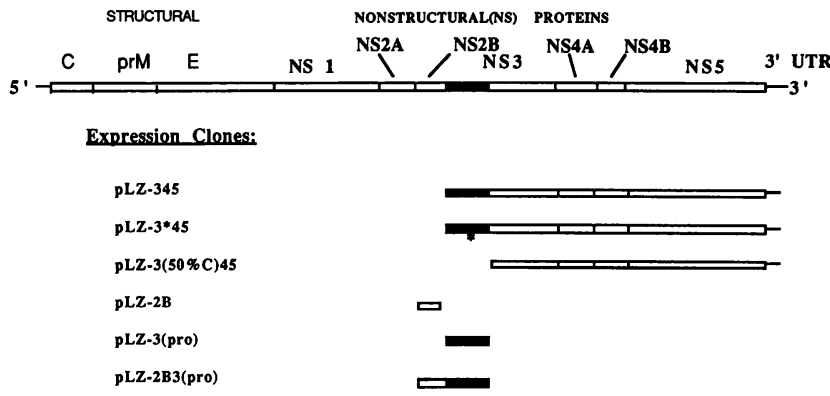

FIG. 1. Plasmids encoding DEN-2 nonstructural proteins and their precursors used for the infection-transfection protocol. In the top of the figure, a long horizontal bar indicates the open reading frame encoding the DEN-2 precursor polyprotein, and the vertical lines within the bar denote the boundaries of individual mature proteins which result from the processing of the precursor protein. The gene order of the precursor protein consists of the structural region encoding the $\mathrm{C}$, prM, and $\mathrm{E}$ proteins and the nonstructural region which encodes NS1, NS2A, NS2B, NS3, NS4A, NS4B, and NS5 proteins (see reference 4 for a review). Single lines extending from the bar toward the $5^{\prime}$ and $3^{\prime}$ ends denote the untranslated region (UTR) of the DEN-2 RNA. The sizes are not drawn to scale. The pLZ series of plasmids indicate the locations of the coding sequences of the various nonstructural proteins and their precursors used in this study. The experimental details of the plasmid constructs are given in the text. *, mutation of His $\rightarrow$ Ala in the active site of the protease domain of the NS3 protein, which is indicated by a black box.

fragment from pLZ-345. The resulting plasmid, pLZ-3*45, contained the complete NS3 to NS5 coding sequence with a mutation which changed histidine into alanine at the conserved active site catalytic triad of the NS3 protease domain. All PCR-derived clones were verified by sequence analysis.

To examine the processing, we first infected monkey kidney (CV-1) cells with the recombinant vaccinia virus vTF7-3 to provide T7 RNA polymerase $(9,18)$; this was followed by transfection of an expression plasmid by a protocol based on liposome-mediated DNA uptake. This transient expression system is referred to as an infectiontransfection protocol (18). The expression plasmid pLZ-345 gave rise to a $180-\mathrm{kDa}$ protein in the transfected $\mathrm{CV}-1$ cells which could react with either the anti-NS3 or anti-NS5 antibodies, whereas no cleavage of the precursor NS3NS4A-NS4B-NS5 (hereafter referred to as NS345) was observed (data not shown), although the $\mathrm{N}$-terminal region of NS3 contains a protease domain (10). Yellow fever virus (YF) precursor NS345 356 containing one-third of the NS5 protein (3) and the dengue virus type 4 (DEN-4) precursor NS345 (2) also did not undergo processing when expressed alone. The failure of the polyprotein precursor NS345 to undergo processing suggested that the catalytically active NS3 alone was not sufficient for cleavages at the NS3-NS4A and NS4B-NS5 sites and that one or more nonstructural proteins were also required. NS2B was a good candidate for that role because of the recent reports that NS2B is required for NS3 protease activity $(2,3,6,8,22)$. To test whether NS2B could mediate processing of NS345 in trans, we cotransfected an expression clone encoding NS2B with the plasmid encoding NS345. When the labeled cell lysates from the transfected cells were analyzed by immunoprecipitation, it was found that the NS345 polyprotein precursor underwent processing as indicated by the release of NS3 and NS5 (Fig. 2A and B, lanes 2), which would require cleavages at both the NS3-NS4A and NS4B-NS5 sites. These data indicate that NS2B plays an important role in the processing of NS345 at both the NS3-NS4A and NS4B-NS5 sites. How-

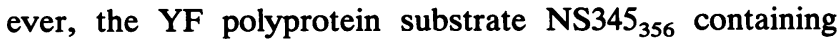
one-third of the NS5 protein was processed by NS2B supplied in trans only at the NS4B-NS5 site, and no detectable cleavage of the NS3-NS4A site was observed (3). In contrast, the DEN-2 NS345 substrate used in our study contains the entire NS5 sequence and could undergo cleavages at both the NS3-NS4A and NS4B-NS5 sites (Fig. 2A and B). From these results, an intact NS5 sequence seems to be important for cleavage at the NS3-NS4A site by NS2B. We found that the cleavage at the NS3-NS4A site is exquisitely sensitive to conformation of the polyprotein precursor; the most efficient cleavage, similar to the pattern in DEN-2infected cells, was observed when the precursor NS12345 containing the entire nonstructural region was used as the substrate (29). Thus, it appears that conformation of a precursor protein may play an important role in the processing events mediated by a viral protease. To investigate whether the NS3 protease domain was important for the processing of NS345, we made a deletion mutant, pLZ$3(50 \% \mathrm{C}) 45$, which encodes a region starting from the C-terminal half of NS3 and ending with the entire NS5 sequence (Fig. 1). Transient expression from this plasmid gave rise to the unprocessed NS3(50\% C terminal) 45 precursor (Fig. 2C, lane 2). Cotransfection of the plasmids encoding NS2B alone or the NS3 protease domain alone [NS3(pro)] could not mediate the processing of the NS3(50\% C terminal) 45 precursor (Fig. 2C, lanes 3 and 4, respectively). But when the plasmids encoding NS2B and NS3(pro) were cotransfected with the precursor plasmid pLZ-3(50\%C) 45, the NS4B-NS5 site of the precursor was cleaved, as shown by the release of NS5 (Fig. 2C, lane 5). Cotransfection of the plasmid encoding the linked NS2B-NS3(pro) sequence also gave a similar result (Fig. 2C, lane 6). However, cleavage of the NS3NS4A site was not detectable in these experiments (data not shown). Two possible explanations could be invoked from this result: the conformation assumed by precursor $\mathrm{A}$ having a large deletion in the NS3 sequence might preclude cleavage at the NS3-NS4A site, or the cis linkage of the NS3 protease domain to NS4A is required for this cleavage, which is abrogated in precursor $\mathrm{A}$. To address this question, we made a mutant plasmid, pLZ-3*45, in which the conserved protease triad residue His- 51 was changed to Ala by site-directed point mutagenesis by PCR (31). An additional mutation (His-47 $\rightarrow$ Asn), the origin of which was traced to the primer oligonucleotide used for PCR, was also found to be present in three independent clones by sequence analysis. Cotransfection of this plasmid with pLZ-2B showed that, unlike the processing of the wild-type precursor (Fig. 2A and B, lanes 2), NS2B alone in trans was ineffective in the processing of this mutant precursor NS3*45 and required coexpression of wild-type NS3(pro) (Fig. 2A and B, lanes 3 and 4). These experiments clearly indicated that the cis linkage of the catalytically active protease domain of NS3 to the NS4A site is not required for the cleavage at the NS3-NS4A site, as it could be cleaved in trans by NS2B and NS3(pro).

The unprocessed precursors as well as the intermediates formed in the processing of NS345 or NS3*45 are identifiable by using anti-NS3 and anti-NS5 antibodies. For example, the intermediate NS3-NS4A was detected by using anti-NS3 antibodies in the processing of the NS345 and NS3*45 precursors (Fig. 2A, lanes 2 and 4). The identity of this band as to NS3-NS4A was confirmed by using additional expression constructs (29). Formation of an NS3-NS4A intermedi- 


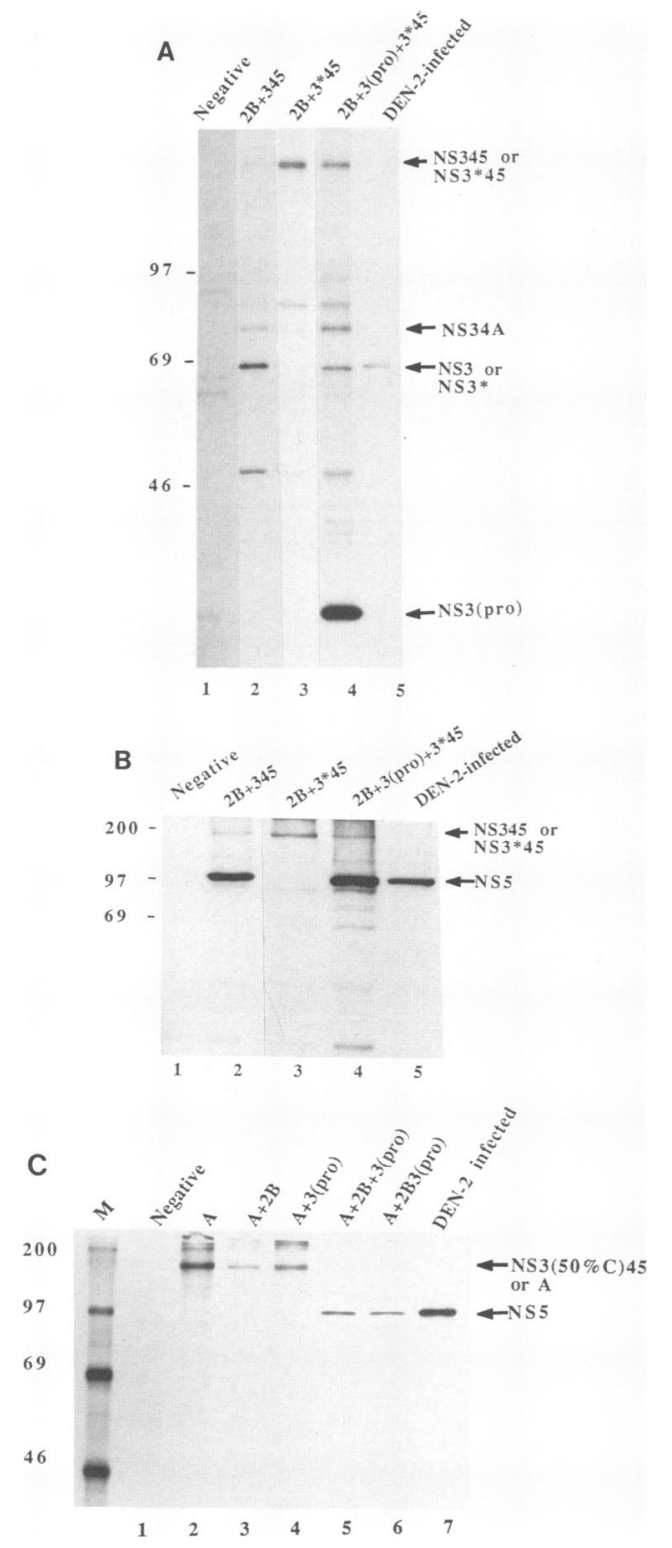

FIG. 2. Processing of the NS345 precursor protein requires NS2B and a catalytically active protease domain. Monolayers of CV-1 cells in $25-\mathrm{cm}^{2}$ flasks were infected with recombinant vaccinia virus expressing T7 RNA polymerase (vTF7-3), except one indicated as DEN-2-infected, and then transfected with the expression plasmid. Three hours posttransfection, the cells were metabolically labeled with $\left.{ }^{35} \mathrm{~S}\right]$ methionine for 4 to $5 \mathrm{~h}$, and the cell lysates were immunoprecipitated with the polyclonal antibodies raised against the intact NS3 and NS5 proteins (29) and analyzed by sodium dodecyl sulfate-polyacrylamide gel electrophoresis and autoradiography. (A) Rabbit polyclonal anti-NS3 antibody was used for immunoprecipitations. The numbers on the left margin denote the sizes of the protein standards in kilodaltons. Lanes: 1, negative control (infection only; no DNA transfection); 2 , plasmids pLZ-2B and pLZ-345 were transfected; 3 , plasmids pLZ-2B and pLZ-3*45 were transfected; 4 , plasmids pLZ-2B, pLZ-3(pro), and pLZ-3*45 were transfected; $5, \mathrm{DEN}-2$ virus-infected CV-1 cell lysates. (B) The samples were the same as in panel A except that the immunoprecipitates were derived from using rabbit polyclonal anti-NS5 antibody. (C) The immunoprecipitates were derived from using rabbit polyclonal anti-NS5 antibody. Lanes: $\mathrm{M},{ }^{14} \mathrm{C}$-labeled protein molecular mass standards (kilodaltons); 1 , negative control; 2, plasmid pLZ-3(50\%C)45 (referred to as A) was transfected; 3, plasmids A ate reactive with anti-NS3 antibodies is one evidence that the cleavage of NS4A-NS4B had taken place in the processing of NS345. Since specific antibodies against these dengue viral antigens, NS4A and NS4B, are not presently available, we could not confirm independently the cleavage of the NS4A-NS4B site by immunoprecipitation. However, the efficiency of cleavage at the NS3-NS4A site of the precursor NS345 or NS3*45 can be estimated from the ratio of the mature NS3 (or the NS3*) to the unprocessed precursor NS345 (or NS3*45) and the amount of the intermediate NS3-NS4A formed (Fig. 2A, lanes 2 and 4); this efficiency was better with the wild-type NS345 substrate, in which the catalytically active NS3 protease domain was linked in cis to NS4A and when only NS2B was supplied in trans (Fig. 2A, lane 2), than with the processing of the mutant NS3*45 substrate, in which both the active NS3(pro) and NS2B were supplied in trans. This observation may be explained by the possibility that the NS3-NS4A site can be cleaved in both cis and trans configurations during the processing of the wildtype substrate NS345; once a small fraction of the mature NS3 is released by cis cleavage, the rest of NS3 can be released from the precursor NS345 by both cis and trans cleavages at the NS3-NS4A site. However, the cleavage of the NS3-NS4A site in the NS3*45 precursor by NS2B and NS3(pro) can occur only in the trans configuration. This difference in the efficiency of cleavage at the NS3-NS4A site was not observed for the cleavage at the NS4B-NS5 site of the two substrates, as the intermediate NS4B-NS5 was not formed at a level detectable with anti-NS5 antibodies (Fig. 2B, lanes 2 and 4). Although the NS4B-NS5 site can be cleaved in trans mediated by NS2B and NS3(pro), we cannot rule out the possibility that in the natural processing of the DEN-2 polyprotein, a cis cleavage is involved if the catalytically active NS3(pro) domain in conjunction with NS2B is brought in close proximity to the NS4B-NS5 site (see the model in Fig. 4). Experiments are in progress to clarify this aspect.

It is not yet understood what role NS2B plays in conjunction with NS3 in the processing of the precursor protein. NS3 by itself may not have the right conformation for protease activity, and it is possible that the interaction of NS2B with NS3 confers protease activity for NS3. In this regard, the proteolytic processing done by the NS2B and NS3 proteins resembles the two-component protein requirement for the cleavage of amino-terminal targeting sequences in the mitochondrial matrix of Neurospora crassa (13): the 57-kDa matrix processing peptidase having the catalytic site and the $52-\mathrm{kDa}$ processing enhancing protein. Matrix processing peptidase alone was shown to have a low processing activity, whereas processing enhancing protein alone was devoid of any activity, but it significantly enhanced the processing activity of matrix processing peptide. It is unknown at present whether NS3 alone has any basal protease activity in the absence of NS2B. It is possible that the system used in this study may not be sensitive enough to detect the low protease activity, if any, of NS3. Studies using model substrates containing the dibasic cleavage sites with sensitive detection methods may address this question.

We then examined the subcellular localization of the

and pLZ-2B were transfected; 4, plasmids $A$ and pLZ-3(pro) were transfected; 5, plasmids A, pLZ-2B, and the pLZ-3(pro) were transfected; 6, plasmids $A$ and pLZ-2B3(pro) were transfected; 7, DEN-2-infected cell lysates. 

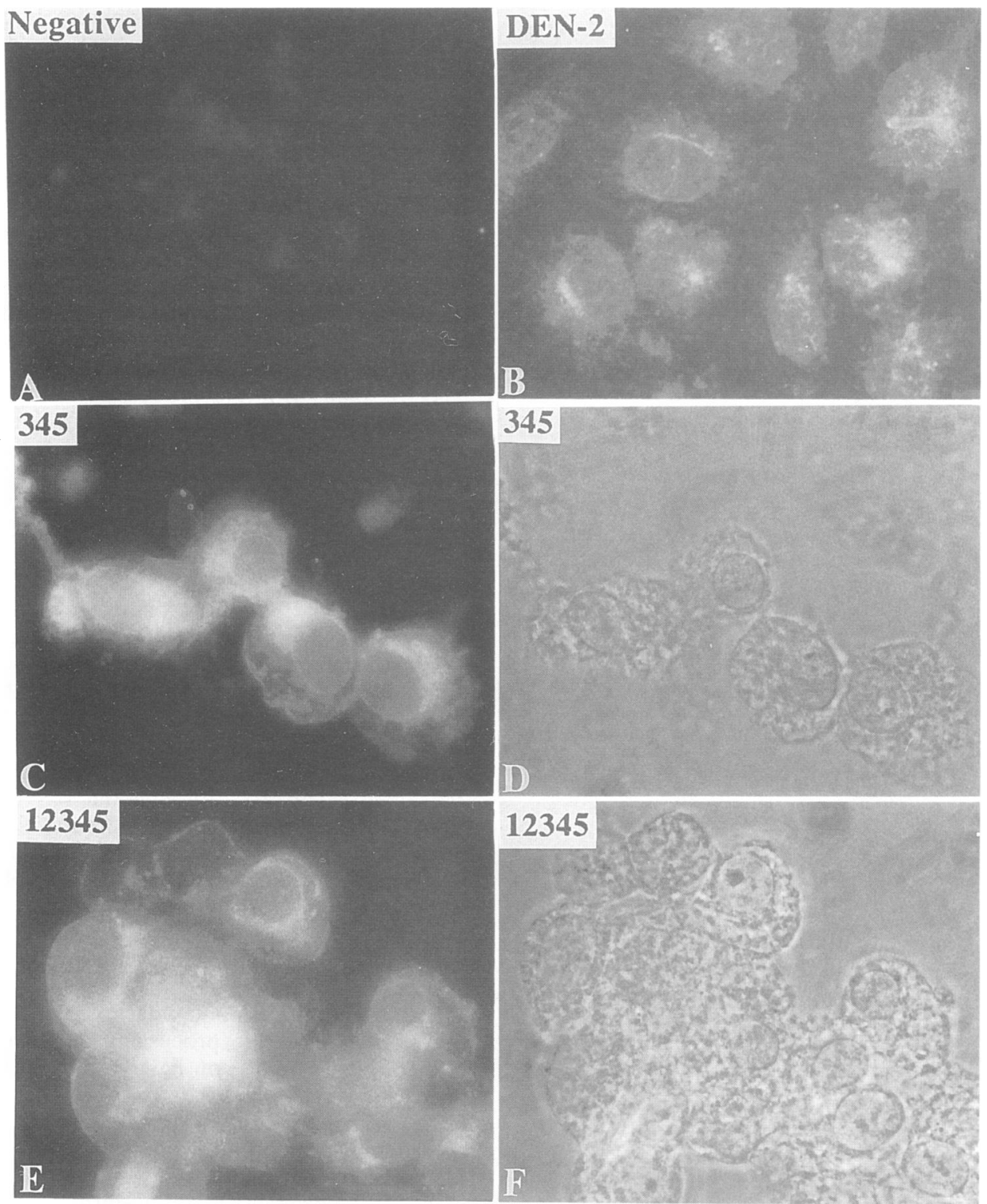

FIG. 3. Subcellular localization of the unprocessed precursor NS345. CV-1 cells were used for the infection-transfection protocol. The cells were infected with the VTF7-3 virus (1 PFU per cell) at room temperature for $30 \mathrm{~min}$. Subsequently, the vTF7-3-infected cells were transfected with the indicated plasmid for $3 \mathrm{~h}$. Cells were then incubated with Dulbecco modified Eagle medium containing 5\% fetal bovine serum for about $12 \mathrm{~h}$. Cells were fixed for $30 \mathrm{~min}$ with paraformaldehyde (3.7\%) in phosphate-buffered saline (PBS) (pH 7.4) and then treated with Triton X-100 (0.1\%) in PBS for $10 \mathrm{~min}$. Cells were then washed with PBS for $30 \mathrm{~min}$ and prepared for indirect immunofluorescence with anti-NS5 antibodies as previously described (30). (A) vTF7-3-infected cells as the negative control. (B) CV-1 cells were infected with DEN-2 virus at $1 \mathrm{PFU}$ per cell for $1 \mathrm{~h}$ at room temperature. Then the cells were incubated with Dulbecco modified Eagle medium containing $10 \%$ fetal bovine serum for $48 \mathrm{~h}$ before being fixed for immunofluorescence. (C) vTF7-3-infected cells were transfected with pLZ-345. (D) Phase-contrast picture of cells in panel C. (E) Same as panel C, except transfected with pLZ-12345. (F) Phase-contrast picture of cells in panel E.

polyprotein precursor NS345 by indirect immunofluorescence with rabbit polyclonal antibodies against purified NS3 and NS5. We found that the unprocessed precursor was associated with some subcellular organelle in the cytoplasm of the majority of cells transfected with the plasmid pLZ-345 (Fig. 3C), whereas the vTF7-3-infected cells alone were negative in immunoreactivity with anti-NS5 antibodies (Fig. 3A). However, in the cells transfected with the plasmid pLZ-12345 in which the precursor was processed efficiently
(29), the released NS5 was localized predominantly in the perinuclear region, although some diffuse cytoplasmic distribution was also seen (Fig. 3E and F). It is interesting that flavivirus replication sites were also localized in the perinuclear region by using antibodies to a double-stranded RNA and autoradiographic and immunofluorescence techniques $(16,20,27)$. NS5 has been implicated in the replication of the flavivirus genome (12). In the DEN-2-infected CV-1 cells (Fig. 3B), NS5 was seen to be distributed in the perinuclear 
region, although some cytoplasmic and nuclear staining was also seen (Fig. 3B). Thus, our results suggest that the processing of the polyprotein precursor occurs at an intracellular site distinct from the site of localization of the processed NS5. In fact, in Sindbis virus, a member of the alphaviruses, it was shown that the precursor of the structural proteins and the cleavage products were destined to different compartments (reviewed in reference 15). For example, the mature capsid protein cleaved off from the amino terminus of the precursor assembled in the cytoplasm, whereas the C-terminal portion of this precursor is transported to the rough endoplasmic reticulum for further processing.

The precursor NS2B345 polyprotein could not be detected in the YF-infected cells (5), probably due to a rapid cleavage of the NS2B-NS3 site by the NS3 protease. This cleavage event would give rise to the precursor NS345 as a transient intermediate, the processing of which must await the trans function of NS2B at the NS3-NS4A and NS4B-NS5 sites. The transient existence of this precursor has been noted in YF-infected cells by pulse-chase experiments (5) and in DEN-2-infected cells (data not shown). Two points drawn from the present experimental evidence suggest that the precursor NS345 may assume a special conformation prior to its processing into mature products. First, the NS4ANS4B site could not be cleaved in the DEN-2 precursor NS345 (this study), in NS4A-NS4B (21), in the DEN-4 precursor NS345, or in the YF precursor NS345 ${ }_{356}$ containing the truncated NS5 $(2,3)$; but this site could be cleaved in NS34A/4B(84\%) and NS4A4B5 $(2,8)$. Second, the NS345 precursor has several consensus cleavage sites, although actual cleavage occurs only at the NS3-NS4A and NS4BNS5 sites.

The nonstructural proteins NS4A and NS4B consist of hydrophobic membrane-spanning domains, and the $\mathrm{N}$-terminal amino acid sequence data for Kunjin virus (KUN) and YF suggest that the cleavage of the NS4A-NS4B site may occur in the lumen of the rough endoplasmic reticulum. NS4B has been identified in KUN- and YF-infected cells (5, 24), whereas NS4A has been identified only in KUN-infected cells (25). On the basis of our present knowledge regarding DEN-2 polyprotein processing, we propose a model for the conformation of the NS345 precursor (Fig. 4). In this model, the NS4A-NS4B region subsequent to insertion into the endoplasmic reticulum membrane might confer a cytoplasmic anchor for the precursor NS345 and thus account for its localization seen in Fig. 3C. In the absence of NS2B, the localization of NS345 may be confined to this cytoplasmic organelle.

However, the polyprotein precursor NS12345 undergoes processing mediated by NS2B linked in cis, and the released NS5 was localized to the perinuclear region (Fig. 3B). Assembly of the replication complexes at the perinuclear region would require the localization of the genomic RNA at this site to serve as a template for negative-strand synthesis (for reviews, see references 1,26 , and 27). It is not clear how the timing of the translation of the positive-strand RNA genome into mature proteins and of its replication to progeny RNA molecules is controlled. It is possible that the translation of the genomic RNA must precede its replication in order to produce the nonstructural proteins required for replication. NS3 has been implicated to play a dual role as the protease as well the RNA helicase based on the presence of conserved domains in the region $\mathrm{C}$ terminal to the protease domain (11). NS5 protein has been implicated to function as an RNA polymerase based on the presence of the

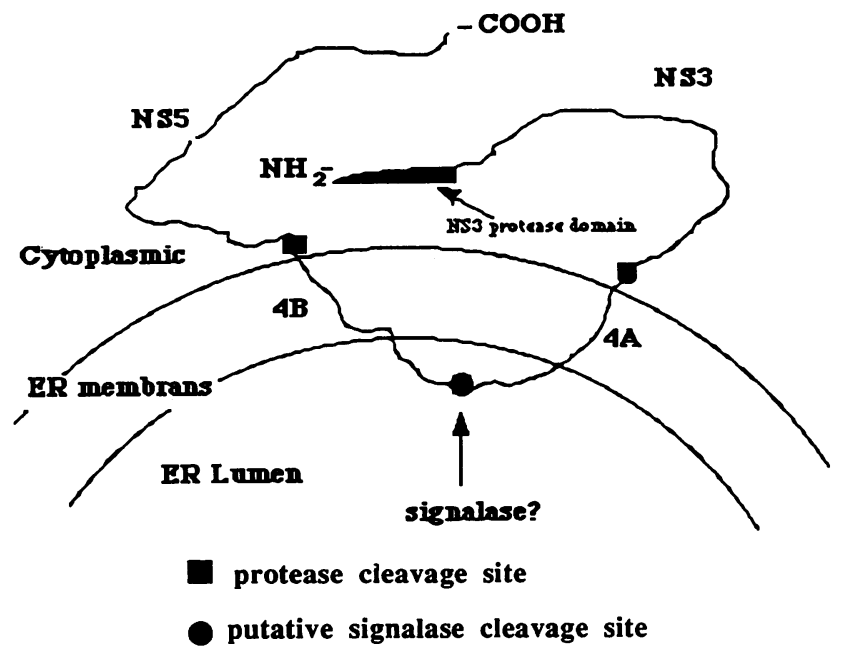

FIG. 4. Proposed model for the conformation of the unprocessed NS345 precursor. The precursor is localized in the endoplasmic reticulum (ER) membrane because of the hydrophobic sequences present in NS4A and NS4B. The NS3 protease domain is indicated by an arrow.

highly conserved G-D-D motif found in RNA-dependent RNA polymerases of several positive-strand RNA viruses. It is possible that the transient formation of the precursor NS345 and subsequent processing to give rise to mature NS3 and NS5 might serve as a control point during the switch from the translation mode of the viral RNA template into its replication mode.

We thank Bernard Moss for his gift of the vaccinia virus vector plasmid (pTM1) and the vTF7-3 virus used in this study, Charles Rice and James Strauss for their gifts of antibodies against NS5 and NS3 proteins, respectively, and Robert Putnak for the DEN-2 hyperimmune ascitic fluid and the monoclonal antibody against NS1 (3E9). We thank T. Trirawatanapong for help with the DEN-2 infections of CV-1 cells and M. Ramachandra and M. Kapoor for critical reading of the manuscript.

This work was partly supported by a contract from the U.S. Army Medical Research and Development Command (DAMD17-85-C5273) to R.P.

\section{REFERENCES}

1. Brinton, M. A. 1986. Replication of flaviviruses, p. 327-365. In S. Schlesinger and M. J. Schlesinger (ed.), The Togaviridae and Flaviviridae. Plenum Press, New York.

2. Cahour, A., B. Falgout, and C.-J. Lai. 1992. Cleavage of the dengue virus polyprotein at the NS3/NS4A and NS4B/NS5 junction is mediated by viral protease NS2B-NS3, whereas NS4A/NS4B may be processed by a cellular protease. J. Virol. 66:1535-1542.

3. Chambers, T. J., A. Grakoui, and C. M. Rice. 1991. Processing of the yellow fever virus nonstructural polyprotein: a catalytically active NS3 protease domain and NS2B are required for cleavages at dibasic sites. J. Virol. 65:6042-6050.

4. Chambers, T. J., C. S. Hahn, R. Galler, and C. M. Rice. 1990. Flavivirus genome organization expression, and replication. Annu. Rev. Microbiol. 44:649-688.

5. Chambers, T. J., D. W. McCourt, and C. M. Rice. 1990. Production of yellow fever virus proteins in infected cells: identification of discrete polyprotein species and analysis of cleavage kinetics using region-specific polyclonal antisera. Virology 177:159-174.

6. Chambers, T. J., R. C. Weir, A. Grakoui, D. W. McCourt, J. F. Bazan, R. J. Fletterick, and C. M. Rice. 1990. Evidence that the $\mathrm{N}$-terminal domain of nonstructural protein NS3 from yellowfe- 
ver virus is a serine protease responsible for site-specific cleavage in the viral polyprotein. Proc. Natl. Acad. Sci. USA 87:8898-8902.

7. Elroy-Stein, O., T. R. Fuerst, and B. Moss. 1989. Cap-independent translation of mRNA conferred by encephalomyocarditis virus $5^{\prime}$ sequence improves the performance of the vaccinia virus/bacteriophage T7 hybrid expression system. Proc. Natl. Acad. Sci. USA 86:6126-6130.

8. Falgout, B., M. Pethel, Y. Zhang, and C.-J. Lai. 1991. Both nonstructural proteins NS2B and NS3 are required for the proteolytic processing of dengue virus nonstructural proteins. J. Virol. 65:2467-2475.

9. Fuerst, T. R., E. G. Niles, W. Studier, and B. Moss. 1986. Eukaryotic transient-expression system based on recombinant vaccinia virus that synthesizes bacteriophage T7 RNA polymerase. Proc. Natl. Acad. Sci. USA 83:8122-8126.

10. Gorbalenya, A. E., A. P. Donchenko, E. V. Koonin, and V. M. Blinov. 1989. N-terminal domains of putative helicases of flaviand pestiviruses may be serine proteases. Nucleic Acids Res. 17:3889-3897.

11. Gorbalenya, A. E., E. V. Koonin, A. P. Donchenko, and V. M. Blinov. 1989. Two related superfamilies of putative helicase involved in replication, recombination, repair and expression of DNA and RNA genomes. Nucleic Acids Res. 17:4713-4729.

12. Grun, J. B., and M. A. Brinton. 1987. Dissociation of NS5 from cell fractions containing West Nile virus-specific polymerase activity. J. Virol. 61:3641-3644.

13. Hawlitschek, G., H. Schneider, B. Schmidt, M. Tropschug, F.-U. Hartl, and W. Neupert. 1988. Mitochondrial protein import: identification of processing peptidase and of PEP, a processing enhancing protein. Cell 53:795-806.

14. Irie, K., P. M. Mohan, Y. Sasaguri, R. Putnak, and R. Padmanabhan. 1989. Sequence analysis of cloned dengue virus type 2 genome (New Guinea-C strain). Gene 75:197-211.

15. Krausslich, H.-G., and E. Wimmer. 1988. Viral proteinases. Annu. Rev. Biochem. 57:701-754.

16. Lubiniecki, A. S., and C. J. Henry. 1974. Autoradiographic localization of RNA synthesis directed by arboviruses in the cytoplasm of infected BHK-21 cells. Proc. Soc. Exp. Biol. Med. 145:1165-1169.

17. Maniatis, T., E. F. Fritsch, and J. Sambrook. 1982. Molecular cloning: a laboratory manual. Cold Spring Harbor Laboratory, Cold Spring Harbor, N.Y.
18. Moss, B., O. Elroy-Stein, T. Mizukami, W. A. Alexander, and T. R. Fuerst. 1990. New mammalian expression vectors. Nature (London) 348:91-92.

19. Nakano, R., L.-J. Zhao, and R. Padmanabhan. 1991. Overproduction of adenovirus DNA polymerase and preterminal protein in HeLa cells. Gene 105:173-178.

20. Ng, M. L., J. S. Pederson, B. H. Toh, and E. G. Westway. 1983. Immunofluorescent sites in Vero cells infected with the flavivirus Kunjin. Arch. Virol. 78:177-190.

21. Preugschat, F., and J. H. Strauss. 1991. Processing of nonstructural proteins NS4A and NS4B of dengue 2 virus in vitro and in vivo. Virology 185:689-697.

22. Preugschat, F., C. W. Yao, and J. H. Strauss. 1990. In vitro processing of dengue virus type 2 nonstructural proteins NS2A, NS2B, and NS3. J. Virol. 64:4364-4374.

23. Rice, C. M., E. G. Strauss, and J. H. Strauss. 1986. Structure of the flavivirus genome, p. 279-326. In S. Schlesinger and M. J. Schlesinger (ed.), The Togaviridae and Flaviviridae. Plenum Press, New York.

24. Speight, G., G. Coia, M. D. Parker, and E. G. Westaway. 1988. Gene mapping and positive identification of the nonstructural proteins NS2A, NS2B, NS3, NS4B, and NS5 of the flavivirus Kunjin and their cleavage sites. J. Gen. Virol. 69:23-34.

25. Speight, G., and E. G. Westaway. 1989. Positive identification of NS4A, the last of the hypothetical nonstructural proteins of flaviviruses. Virology 170:299-301.

26. Westaway, E. G. 1980 . Replication of flaviviruses, p. 531-581. In R. W. Schlesinger (ed.), The togaviruses: biology, structure, and replication. Academic Press, Inc., New York.

27. Westaway, E. G. 1987. Flavivirus replication strategy. Adv. Virus Res. 33:45-90.

28. Westaway, E. G., M. A. Brinton, S. Y. Gaidamovich, M. C. Horzinek, A. Igarashi, L. Kaariainen, D. K. Lvov, J. S. Porterfield, P. K. Russell, and D. W. Trent. 1985. Flaviviridae. Intervirology 24:183-192.

29. Zhang, L., and R. Padmanabhan. Unpublished data.

30. Zhao, L.-J., and R. Padmanabhan. 1988. Nuclear transport of adenovirus DNA polymerase is facilitated by interaction with preterminal protein. Cell 55:1005-1015.

31. Zhao, L.-J., Q.-X. Zhang, and R. Padmanabhan. 1992. Polymerase chain reaction-based point mutagenesis protocol. Methods Enzymol. 217:218-227. 